\title{
$\mathrm{OFDM}$ 시스템에서 비중복 프리코딩을 이용한 미상 채널 추정 방법
}

정회원 서 방 원

\section{Non-redundant Precoding Based Blind Channel Estimation Scheme for OFDM Systems}

\author{
Bangwon Seo* Regular Member
}

요 약

\begin{abstract}
직교 주파수 분할 다중화 시스템에서 비중복 프리코딩을 이용한 미상 채널 추정 방식을 제안한다. 제안한 방식 에서는 수신 신호에 대한 공분산 행렬을 구하고, 그 행렬의 각 원소들을 프리코딩 행렬의 각 원소로 나눔으로써 변형된 공분산 행렬을 구한다. 이 행렬의 최대 고유값에 해당하는 고유벡터를 구함으로써 채널 계수들을 추정하게 된다. 이 때, 고유 벡터를 구하기 위하여 많은 계산량을 필요로 하는 고유치 분해 기법 대신에 간단한 파워 기법 을 적용함으로써 계산량을 크게 줄이게 된다. 제안하는 채널 추정 방식의 평균 제곱 오차에 대한 이론적인 값을 유도하고, 모의실험 결과와 비교함으로써 유도한 값이 실험 결과와 일치한다는 것을 확인한다. 또한, 모의실험을 통해서, 제안한 방법이 기존 방법들보다 더 우수한 채널 추정 성능과 비트 오율 성능을 나타낸다는 것을 보인다.
\end{abstract}

Key Words : OFDM, channel estimation, precoding, 채널 추정, 프리코딩

\section{ABSTRACT}

For orthogonal frequency-division multiplexing (OFDM) systems, we propose a blind channel estimation scheme based on non-redundant precoding. In the proposed scheme, a modified covariance matrix is first obtained by dividing the covariance matrix of the received signal vector by the precoding matrix element-by-element. Then, the channel vector is estimated as an eigenvector corresponding to the largest eigenvalue of the modified covariance matrix. The eigenvector can be obtained by power method with low computational complexity instead of the complicated eigenvalue decomposition. We analytically derive a mean square error (MSE) of the proposed channel estimation scheme and show that the analysis result coincides well with the simulation result. Also, simulation results show that the proposed scheme has better MSE and bit error rate (BER) performance than conventional channel estimation schemes.

\section{I. 서 론}

직교 주파수 분할 다중화 (Orthogonal frequency-division multiplexing: OFDM) 기술은 높
은 주파수 효율성, 주파수 선택적 페이딩에 대한 강 인성, 송수신기 구조의 단순성과 같은 좋은 특징들 을 가지고 있기 때문에, 고속 데이터 통신을 필요로 하는 무선 통신 시스템에 적합한 기술로 주목 받고

* 한국전자통신연구원 차세대통신연구부문 이동통신방식연구팀(seobw@etri.re.kr)

논문번호 : KICS2012-02-102, 접수일자 : 2012년 2월 29일, 최종논문접수일자 : 2012년 5월30일 
있다. OFDM 기술은 유럽의 디지털 오디외비디오 방송 시스템 (Digital audio/video broadcasting system: DAB, DVB)에서 사용되고 있으며, 또한 IEEE 802.11a, IEEE $802.11 \mathrm{~g}$ 와 같은 고속 무선 랜 시스템에서도 사용되고 있다. 또한, 최근에 4세대 이동통신 시스템으로 주목 받고 있는 3GPP LTE 시스템과 IEEE $802.16 \mathrm{~m}$ 시스템에서도 이 기술이 사용되고 있다.

고속 데이터 통신이 제대로 동작하기 위해서는 채널 환경에 따른 데이터의 적응적 로딩 (Adaptive loading), 응집 검파 (Coherent detection)와 같은 기 술들을 필요로 하며, 이를 위해서는 적절한 채널 추 정기를 통해서 채널 값을 미리 알고 있어야 한다. 채널 추정 방법들은 훈련 심볼 (Pilot symbol)의 전 송 유무에 따라서 훈련 심볼 기반의 채널 추정 방 법과 미상 (Blind) 채널 추정 방법으로 나눌 수 있 다. 훈련 심볼 기반의 채널 추정 방법은 일반적으로 미상 채널 추정 방법보다 성능이 우수하고 계산량 이 간단하지만, 훈련 심볼을 주기적으로 전송해야 되기 때문에 주파수 효율이 떨어지는 단점이 있다. 반면에 미상 추정 방법은 채널 추정을 위한 훈련 심볼들을 전송할 필요가 없기 때문에 매우 좋은 주 파수 효율성을 가지며, 이로 인해 많은 관심을 받아 왔다.

OFDM 시스템에서 제안된 대부분의 미상 채널 추정 방법들은 먼저 수신 신호에 대한 공분산 행렬 을 구하고, 이것으로부터 잡음 성분에 대한 부공간 (Subspace)을 나타내는 행렬을 구한다 ${ }^{[1,2]}$. 그리고 나서, 이 잡음 부공간 행렬과 신호 벡터 성분 간의 직교성을 이용하여 채널 벡터를 추정하게 된다. 이 방법들은 공분산 (Covariance) 행렬에 대한 고유값 분해 (Eigenvalue decomposition)를 필요로 하고, 공분산 행렬의 길이는 부반송파의 개수와 같거나 또는 그것보다 더 큰 값을 갖는다. 따라서, 공분산 행렬에 대한 고유값 분해 과정은 매우 복잡한 계산 을 필요로 한다.

최근에 비중복 프리코딩 (Non-redundant precoding) 행렬을 사용함으로써 부반송파 심볼들 간에 상관성 (Correlation)을 갖도록 만들고 이를 이 용하여 채널을 추정하는 미상 채널 추정 방법들이 제안되었다 ${ }^{[3-7]}$. 여기에서는 비중복 프리코딩을 적용 하기 때문에 데이터 전송 효율이 그대로 유지되는 장점이 있다. [3], [4]에서는 복잡한 고유값 분해 과 정을 필요로 하지 않고 단순히 비중복 프리코딩 행 렬의 구조와 수신 신호 공분산 행렬을 이용하는 미
상 채널 추정 방법들을 제안하였다. 그러나, 이러한 방법들은 백색 부가 가우시안 잡음 (Additive white Gaussin noise: AWGN)의 영향을 무시하기 때문에, $\mathrm{AWGN}$ 의 세기가 다른 간섭들보다 상대적으로 더 큰 영역에서는 이러한 방법들의 성능이 떨어지는 단점이 있다.

$\mathrm{AWGN}$ 의 영향을 줄이기 위하여 [5]에서는 두 개의 연속된 $\mathrm{OFDM}$ 심볼에 걸쳐서 비중복 프리코 딩을 적용하고 두 심볼 간의 수신 신호 교차 상관 행렬을 이용하는 미상 채널 방법을 제안하였다. 그 러나, 이 방법에서는 두 개의 OFDM 심볼에 걸쳐 서 프리코딩을 적용하기 때문에 OFDM 심볼의 개 수가 짝수 개로 존재해야 되고, 또한 여기에서 사용 하는 교차 상관 행렬의 길이가 [3], [4]에서 사용하 는 수신 신호 공분산 행렬의 길이보다 두 배 크기 때문에, 교차 상관 행렬을 추정하기 위해서는 [3], [4]보다 더 많은 수신 심볼들을 필요로 하게 된다.

한편, [6], [7]에서는 AWGN의 영향을 줄이기 위 하여, 수신 신호에 대한 4차 이상의 고차 통계적 특 성 (Higher-order statistics)을 이용하는 독립 성분 분석 (Independent component analysis: ICA) 방식 을 적용하는 방법들은 제안하였다. 그러나, 이 방법 에서는 수신 신호에 백색화 여파기 (Whitening filter)를 적용해야 되고, 이 때 수신 신호에 대한 고 유치 분해를 필요로 하기 때문에, [3]-[5] 방법들보 다 훨씬 더 많은 계산량을 필요로 한다.

본 논문에서는 [3], [4]에서와 마찬가지로 한 개 의 OFDM 심볼에 대해 프리코딩을 적용하고, [3], [4]와 유사한 계산량을 필요로 하면서 $\mathrm{AWGN}$ 의 영 향을 고려하는 미상 채널 추정 기법을 제안한다. 제 안하는 방법에서는, 먼저 수신 신호에 대한 공분산 행렬을 구하고, 그 행렬의 각 원소들을 프리코딩 행 렬의 각 원소들로 나눔으로써 변형된 공분산 행렬 을 구한다. 그리고 나서, 이 공분산 행렬에 대한 최 대 고유값 벡터를 구함으로서 채널 벡터를 추정하 게 된다. 이 때, 최대 고유값 벡터만을 필요로 하기 때문에, 고유값 분해와 같은 복잡한 계산을 필요로 하지 않으며, 파워 기법 (Power method) [8]을 이 용하여 간단하게 최대 고유값 벡터를 구할 수 있다.

본 논문의 구성은 다음과 같다. II장에서는 시스 템 모형을 살펴보고, III장에서는 제안하는 미상 채 널 추정 방법에 대해서 설명한다. IV장에서는 제안 하는 채널 추정 방법에 대한 성능을 이론적으로 분 석한다. V장에서는 모의실험을 통해 이론적 성능 분석 결과가 모의실험 결과와 일치한다는 것을 보 
이고, 또한 제안하는 방법이 기존의 방법들보다 더 우수한 성능을 나타낸다는 것을 보인다. 마지막으로, VI장에서는 결론을 맺는다.

\section{II. 시스템 모형}

먼저, 송신기에서의 과정은 다음과 같다. $k$ 번째 $\mathrm{OFDM}$ 심볼 전송 구간에서의 전송 심볼 블록을 $\mathrm{s}(k)=\left[s_{1}(k), \cdots, s_{N}(k)\right]^{T}$ 로 나타내고, 이것의 평균 과 분산을 각각 $E[\mathbf{S}(k)]=0_{N \times 1}$, $E\left[\mathbf{S}(k) \mathbf{s}(k)^{H}\right]=\mathrm{I}_{N}$ 라고 가정하자. 여기에서 $N$ 은 부 반송파의 개수를 나타내고, $\mathrm{I}_{N}$ 은 길이가 $N \times N$ 인 단위행렬을 나타낸다. 프리코딩에 의한 데이터 전송 률 감소를 발생시키지 않기 위하여 길이가 $N \times N$ 인 선형 비중복 (Non-redundant) 프리코딩을 전송 심볼 블록에 적용한다. 선형 비중복 프리코딩 행렬 을 $\mathrm{P}$ 라고 하고, $N$-포인트 이산 푸리에 역변환 행 렬 (Inverse discrete Fourier transform: IDFT)을 $\mathrm{W}^{H}$ 라고 하면, 송신 신호는 다음과 같이 쓸 수 있 다.

$$
\mathbf{x}(k)=\mathrm{W}^{H} \mathrm{Ps}(k) .
$$

여기에서 $\mathrm{W}$ 의 $(m, n)$ 번째 원소는 $[\mathrm{W}]_{m, n}=\exp \{-j 2 \pi(m-1)(n-1) / N\} / \sqrt{N}$ 이다. 수신 신호에서 부반송파 간 간섭이 발생하지 않도 록 하기 위하여, 이 신호에 순환 전치 (Cyclic prefix) 부분을 추가한 후에 채널을 통해서 전송한 다. 이산 채널 응답 벡터를 길이가 $L$ 인 벡터 $\mathrm{g}=\left[g_{0}, \cdots, g_{L-1}\right]^{T}$ 라고 가정하고, 설명의 편의상 $\|\boldsymbol{g}\|^{2}=1$ 라고 하자. 순환 전치의 길이는 채널의 길 이인 $L$ 보다 크도록 설정되며, 일반적으로 $L$ 은 $N$ 보다 매우 작은 값을 갖는다.

이제 수신기에서의 과정에 대해서 살펴보자. 수 신기에서는 송신기에서 적용한 과정들의 반대 과정 들을 적용한다. 수신기에서 $\mathrm{DFT}$ 를 적용한 후의 결 과 신호는 다음과 같이 쓸 수 있다.

$$
\mathbf{y}(k)=\left[y_{1}(k), \cdots, y_{N}(k)\right]^{T}=\operatorname{HPs}(k)+z(k) .
$$

여기에서 $\mathrm{Z}(k)$ 는 길이가 $N$ 인 $\mathrm{AWGN}$ 으로서, 평 균이 $0_{N \times 1}$ 이고 분산이 $\sigma_{z}^{2} \mathrm{I}_{N}$ 이다. 행렬 $\mathrm{H}=\operatorname{diag}\left(H_{1}, \cdots, H_{N}\right)$ 은 주파수 영역에서의 채널 응 답을 나타내고, $\mathrm{H}$ 와 $\mathrm{g}$ 의 관계는 다음과 같다.

$$
\mathrm{h}=\left[H_{1}, \cdots, H_{N}\right]^{T}=\mathrm{W}_{L} \mathbf{g} .
$$

길이가 $N \times L$ 인 행렬 $\mathrm{W}_{L}$ 은 $\mathrm{W}$ 의 처음 $L$ 개의 열 벡터들로 구성된 행렬이다. 그리고, $\mathrm{h}$ 의 표준값 (Norm)은 $\|\mathrm{h}\|^{2}=\mathrm{g}^{H} \mathrm{~W}_{L}^{H} \mathrm{~W}_{L} \mathrm{~g}=\mathrm{g}^{H} \mathrm{~g}=1$ 이다.

\section{III. 제안하는 미상 채널 추정 방법}

먼저 행렬 $\mathrm{B}$ 를 다음과 같이 정의한다.

$$
\mathrm{B}=\mathrm{PP}^{H} \text {. }
$$

이 때, $\mathrm{B}$ 가 다음과 같은 특성을 갖도록 $\mathrm{P}$ 를 선택 한다.

(특성 1) 모든 $i, j$ 에 대하여 $[\mathrm{B}]_{i, j} \neq 0$.

(특성 2) $[\mathrm{B}]_{1,1}=[\mathrm{B}]_{2,2}=\cdots=[\mathrm{B}]_{N, N}$.

위의 특성들을 만족하는 $\mathrm{P}$ 는 [3] 또는 [4]에서 주어진 방법을 이용하여 쉽게 발생시킬 수 있다.

이제, 수신 신호의 공분산 행렬은 다음과 같이 주어진다.

$$
\mathrm{R}=E\left[\mathbf{y}(k) \mathbf{y}(k)^{H}\right]=\mathrm{HBH}^{H}+\sigma_{z}^{2} \mathrm{I}_{N} .
$$

길이가 $N \times N$ 인 행렬 $\mathrm{Q}$ 를 다음과 같이 정의하 면, $\mathrm{Q}$ 는 변형된 공분산 행렬을 나타낸다.

$$
[\mathrm{Q}]_{m, n}=[\mathrm{R}]_{m, n} /[\mathrm{B}]_{m, n} .
$$

여기에서 $[\mathrm{A}]_{m, n}$ 은 $\mathrm{A}$ 의 $(m, n)$ 번째 원소를 나 타낸다. $\mathrm{H}$ 는 대각행렬이기 때문에, $\mathrm{Q}$ 는 다음과 같 이 표현된다.

$$
\begin{aligned}
\mathrm{Q} & =\left[\begin{array}{cccc}
H_{1} H_{1}^{*}+\tilde{\sigma}_{z}^{2} & H_{1} H_{2}^{*} & \cdots & H_{1} H_{N}^{*} \\
H_{2} H_{1}^{*} & H_{2} H_{2}^{*}+\tilde{\sigma}_{z}^{2} & \cdots & H_{2} H_{N}^{*} \\
\vdots & \vdots & \ddots & \vdots \\
H_{N} H_{1}^{*} & H_{N} H_{2}^{*} & \cdots & H_{N} H_{N}^{*}+\tilde{\sigma}_{z}^{2}
\end{array}\right] \\
& =\mathrm{hh}^{H}+\tilde{\sigma}_{z}^{2} \mathrm{I}_{N} .
\end{aligned}
$$

여기에서 $\tilde{\sigma}_{z}^{2}=\sigma_{z}^{2} /[\mathrm{B}]_{1,1}$ 이다. 식 (3)을 이용하면, $\mathrm{Q}$ 는 다음과 같이 다시 쓸 수 있다. 


$$
\mathrm{Q}=\mathrm{W}_{L} \mathrm{gg}^{H} \mathrm{~W}_{L}^{H}+\tilde{\sigma}_{z}^{2} \mathrm{I}_{N}
$$

그리고, $\mathrm{W}_{L}^{H} \mathrm{~W}_{L}=\mathrm{I}_{N}$ 이므로, 길이가 $L \times L$ 인 행렬 $\mathrm{S}$ 는 다음과 같이 표현된다.

$$
\mathrm{S}=\mathrm{W}_{L}^{H} \mathrm{Q} \mathrm{W}_{L}=\mathrm{gg}^{H}+\tilde{\sigma}_{z}^{2} \mathrm{I}_{L}
$$

이 식으로부터 $\mathrm{g}$ 는 $\mathrm{S}$ 의 신호 부공간 (subspace) 에 존재하는 유일한 신호 벡터라는 것을 알 수 있 으며, $\mathrm{g}$ 와 $\mathrm{S}$ 에 대해서 다음 관계가 성립한다.

$\mathrm{g}=(\mathrm{S}$ 의 최대 고유치에 해당하는 고유 벡터).

또한, $\mathrm{Sg}=\mathrm{g}+\tilde{\sigma}_{z}^{2} \mathrm{~g}=\left(1+\tilde{\sigma}_{z}^{2}\right) \mathrm{g}$ 이므로, $\mathrm{S}$ 의 최대 고 유치는 $\left(1+\tilde{\sigma}_{z}^{2}\right)$ 이다.

이제 식 (12)에서 주어진 정보를 이용하여 $\mathrm{g}$ 에 대한 추정치 $\hat{\mathrm{g}}$ 을 구하는 방법은 다음과 같다.

(단계 A-1) $M$ 개의 수신 신호들을 이용하여 공분산 행렬 $\mathrm{R}$ 을 추정한다.

$$
\widehat{\mathrm{R}}=\frac{1}{M} \sum_{k=1}^{M} \mathrm{y}(k) \mathrm{y}(k)^{H} .
$$

이 때, $M$ 이 커질수록 $\hat{\mathrm{R}}$ 은 $\mathrm{R}$ 에 가까워진다.

(단계 A-2) $\hat{\mathrm{R}}$ 으로부터 $\hat{\mathrm{Q}}, \hat{\mathrm{S}}$ 을 구한다.

$$
\begin{gathered}
{[\hat{\mathrm{Q}}]_{m, n}=[\hat{\mathrm{R}}]_{m, n} /[\hat{\mathrm{B}}]_{m, n} .} \\
\hat{\mathrm{S}}=\mathrm{W}_{L}^{H} \hat{\mathrm{Q}} \mathrm{W}_{L} .
\end{gathered}
$$

(단계 A-3) $\mathrm{g}$ 에 대한 추정치 $\hat{\mathrm{g}}$ 는 다음과 같이 구할 수 있다.

$\hat{\mathrm{g}}=(\hat{\mathrm{S}}$ 의 최대 고유치에 해당하는 고유 벡터) $(16)$

$\hat{\mathrm{S}}$ 에 대한 고유 벡터를 얻기 위하여 $\hat{\mathrm{S}}$ 에 대한 고 유값 분해를 직접 적용하는 것은 $O\left(L^{3}\right)$ 에 해당하 는 계산량을 필요로 한다. 이 때, 일반적으로 $L$ 은 $N$ 보다 매우 작은 값이기 때문에, $\hat{\mathrm{S}}$ 에 대한 고유값 분해는 많은 계산량을 필요로 하지는 않는다. 또한, $\hat{\mathrm{g}}$ 을 추정하기 위해서는 최대 고유치에 해당하는 고 유 벡터만을 필요로 하기 때문에, 고유치 분해 방법 대신 다음과 같은 파워 기법을 적용하면 계산량을 $O\left(L^{2}\right)$ 으로 더욱 줄일 수 있다.
(단계 B-1) 초기화: $j=0$, 임계값 $\mu$ 를 설정, $\mathrm{g}^{(0)}$ 을 초기화한다.

(단계 $\mathrm{B}-2$ ) $j$ 를 1 만큼 증가시키고, 다음을 구한 다.

$$
\begin{gathered}
\mathbf{v}^{(j)}=\hat{\mathbf{S}} \mathbf{g}^{(j-1)} \\
\mathbf{g}^{(j)}=\mathbf{v}^{(j)} /\left\|\mathbf{v}^{(j)}\right\|
\end{gathered}
$$

(단계 B-3) 만약, $\quad\left\|\mathbf{g}^{(j)}-\mathbf{g}^{(j-1)}\right\| /\left\|\mathbf{g}^{(j)}\right\|>\mu$ 이면, 단계 B-2로 이동하고, 그렇지 않으면 $\mathrm{g}$ 에 대한 추정치는 $\hat{\mathrm{g}}=\mathrm{g}^{(j)}$ 에 의해 주어진다.

제안하는 방법에서 가장 많은 계산량을 필요로 하는 부분은 수학식 (13)과 (14)이므로, 제안하는 방법의 계산량은 $O\left(N^{2}\right)$ 이다. 그리고, 기존 방법 [3], [4]의 계산량도 $O\left(N^{2}\right)$ 이다. 따라서, 제안하는 방법은 기존 방법들과 동일한 계산량을 필요로 한 다.

\section{IV. 이론적 성능 분석}

본 장에서는 제안하는 채널 추정 방법에 대한 채 널 추정 오차 성능을 이론적으로 분석한다. 채널 추 정 오차에 대한 평균 제곱 오차 (Mean square error: MSE)를 다음과 같이 정의하자.

$$
\begin{aligned}
\mathrm{MSE} & =E\left[\|\mathrm{~h}-\hat{\mathrm{h}}\|^{2}\right]=E\left[\left\|\mathrm{~W}_{L}(\mathrm{~g}-\hat{\mathrm{g}})\right\|^{2}\right] \\
& =E\left[\|\mathbf{g}-\hat{\mathbf{g}}\|^{2}\right]=E\left[\|\Delta \mathrm{g}\|^{2}\right] .
\end{aligned}
$$

여기에서, $\Delta \mathrm{g}=\mathrm{g}-\hat{\mathrm{g}}$ 이고, $\mathrm{W}_{L}^{H} \mathrm{~W}_{L}=\mathrm{I}_{L}$ 이다.

식 (11)에서 주어진 것처럼 $\mathrm{S}$ 는 한 개의 신호 벡터만을 가지고 있기 때문에, $\mathrm{S}$ 에 대한 고유치 분 해는 다음과 같이 쓸 수 있다 ${ }^{[8]}$.

$$
\begin{aligned}
\mathrm{S} & =\mathrm{gg}^{H}+\tilde{\sigma}_{z}^{2} \mathrm{I}_{L}=\left[\mathrm{u}_{s} \mathrm{U}_{n}\right]\left[\begin{array}{cc}
\Sigma_{s} & 0 \\
0 & \Sigma_{n}
\end{array}\right]\left[\begin{array}{c}
\mathrm{u}_{s}^{H} \\
\mathrm{U}_{n}^{H}
\end{array}\right] \\
& =\Sigma_{s} \mathrm{u}_{s} \mathrm{u}_{s}^{H}+\mathrm{U}_{n} \Sigma_{n} \mathrm{U}_{n}^{H} .
\end{aligned}
$$

여기에서, 길이가 $L \times 1$ 인 벡터 $\mathrm{u}_{s}$ 는 신호 벡터 를 나타내고, 길이가 $L \times(L-1)$ 인 행렬 $\mathrm{U}_{n}$ 은 잡 음 부공간 (Noise subspace)을 나타내는 행렬이다. 따라서, $\mathrm{u}_{s}$ 와 $\mathrm{U}_{n}$ 은 서로 직교하고, 
$\mathrm{u}_{s}^{H} \mathrm{U}_{n}=0_{1 \times(L-1)}, \mathrm{U}_{n}^{H} \mathrm{u}_{s}=0_{(L-1) \times 1}$ 이다. 또한, $\Sigma_{s}$ 는 상수이고, $\Sigma_{n}$ 은 길이가 $(L-1) \times(L-1)$ 인 대 각 행렬이다. 이 때, $\mathrm{g}$ 는 $\mathrm{S}$ 의 신호 부공간에 존재 하는 유일한 고유 벡터이기 때문에, 다음 식이 성립 한다.

$$
\mathrm{u}_{s}=\mathrm{g}, \quad \Sigma_{s}=1+\tilde{\sigma}_{z}^{2} .
$$

유사하게 $\hat{\mathrm{S}}$ 에 대한 고유치 분해는 다음과 같이 쓸 수 있다.

$\hat{\mathrm{S}}=\left[\hat{\mathrm{u}}_{s} \hat{\mathrm{U}}_{n}\right]\left[\begin{array}{cc}\hat{\Sigma}_{s} & 0 \\ 0 & \hat{\Sigma}_{n}\end{array}\right]\left[\begin{array}{c}\hat{\mathrm{u}}_{s}^{H} \\ \hat{\mathrm{U}}_{n}^{H}\end{array}\right]=\hat{\Sigma}_{s} \hat{\mathrm{u}}_{s} \hat{\mathbf{u}}_{s}^{H}+\hat{\mathrm{U}}_{n} \hat{\Sigma}_{n} \hat{\mathrm{U}}_{n}^{H}$.

여기에서, $\hat{\mathrm{u}}_{s}=\hat{\mathrm{g}}$ 이다.

이제 $\hat{\mathrm{Q}}, \hat{\mathrm{u}}_{s}, \hat{\mathrm{U}}_{n}, \hat{\Sigma}_{s}, \hat{\Sigma}_{n}$ 을 각각의 실제 값과 섭동값 (Perturbation)의 합으로 나타내면 다음과 같 다.

$$
\begin{aligned}
& \hat{\mathrm{Q}}=\mathrm{Q}+\Delta \mathrm{Q}, \hat{\mathrm{u}}_{s}=\mathrm{u}_{s}+\Delta \mathrm{u}_{s}, \hat{\mathrm{U}}_{n}=\mathrm{U}_{n}+\Delta \mathrm{U}_{n} \\
& \hat{\Sigma}_{s}=\Sigma_{s}+\Delta \Sigma_{s}, \hat{\Sigma}_{n}=\Sigma_{n}+\Delta \Sigma_{n} . \\
& \mathrm{u}_{s}=\mathrm{g} \text { 이고 } \hat{\mathrm{u}}_{s}=\hat{\mathrm{g}} \text { 이기 때문에, } \Delta \mathrm{g} \text { 는 } \Delta \mathrm{g}=\Delta \mathrm{u}_{s}
\end{aligned}
$$

로 표현할 수 있다. (13)에서 $M$ 이 충분히 크다고 하면, (21)에서 주어진 섭동값들은 실제 값에 비해 서 무시할 정도로 작아진다. 이 경우에는 $\Delta \mathrm{u}_{s}$ 와 $\Delta \mathrm{U}_{n}$ 에 대한 선형 근사값을 얻기 위하여 1 차 섭동 전개식 (1st order perturbation expansion)을 적용할 수 있다 ${ }^{[9]}$.

(제안 명제 1) $M$ 이 충분히 큰 경우에, 제안하는 방식의 채널 추정 오차는 다음과 같이 쓸 수 있다.

$$
\Delta \boldsymbol{g}=\mathrm{U}_{n} \mathrm{U}_{n}^{H}\left(\mathrm{~W}_{L}^{H} \Delta \mathrm{QW} \mathrm{W}_{L}\right) \mathrm{g} .
$$

(증명) $M$ 이 충분히 큰 경우에, 섭동항들 $\Delta \mathrm{u}_{s}$ 와 $\Delta \mathrm{U}_{n}$ 는 각각 $\mathrm{u}_{s}$ 와 $\mathrm{U}_{n}$ 에 비해서 매우 작은 값을 갖고, 1 차 섭동 전개식에 의해서 다음과 같이 근사 화할 수 있다.

$$
\Delta \mathrm{u}_{s}=\mathrm{U}_{n} \mathrm{c}_{1}, \quad \Delta \mathrm{U}_{n}=\mathrm{u}_{s} \mathrm{c}_{2}^{T} .
$$

여기에서, $\mathrm{c}_{1}$ 과 $\mathrm{c}_{2}$ 는 길이가 $(L-1) \times 1$ 인 벡터로
서, 그들의 표준값은 $O(\|\Delta \mathrm{Q}\|)$ 의 범위에 해당한다 [9]. 이제 $\hat{\mathrm{u}}_{s}$ 과 $\hat{\mathrm{U}}_{n}$ 은 다음과 같이 쓸 수 있다.

$$
\hat{\mathrm{u}}_{s}=\mathrm{u}_{s+} \mathrm{U}_{n} \mathrm{c}_{1}, \quad \hat{\mathrm{U}}_{n}=\mathrm{U}_{n}+\mathrm{u}_{s} \mathrm{c}_{2}^{T} .
$$

$\widehat{\mathrm{U}}_{n}^{H} \hat{\mathrm{u}}_{s}=0_{(L-1) \times 1}$ 이므로, 다음을 얻을 수 있다.

$$
\hat{\mathrm{S}} \hat{\mathbf{u}}_{s}=\left(\hat{\Sigma}_{s} \hat{\mathbf{u}}_{s} \hat{\mathbf{u}}_{s}^{H}+\hat{\mathrm{U}}_{n} \hat{\Sigma}_{n} \hat{\mathrm{U}}_{n}^{H}\right) \hat{\mathbf{u}}_{s}=\hat{\Sigma}_{s} \hat{\mathbf{u}}_{s} .
$$

이 식에서 $\hat{\mathrm{u}}_{s}$ 를 (24)로 치환하고, 관계식 $\hat{\mathrm{S}}=\mathrm{W}_{L}^{H}(\mathrm{Q}+\Delta \mathrm{Q}) \mathrm{W}_{L}$ 과 $\hat{\Sigma}_{s}=\Sigma_{s}+\Delta \Sigma_{s}$ 를 이용하면 (25)는 다음과 같이 쓸 수 있다.

$$
\begin{aligned}
& \mathrm{W}_{L}^{H}(\mathrm{Q}+\Delta \mathrm{Q}) \mathrm{W}_{L}\left(\mathrm{u}_{s}+\mathrm{U}_{n} \mathrm{c}_{1}\right) \\
& =\left(\Sigma_{s}+\Delta \Sigma_{s}\right)\left(\mathrm{u}_{s}+\mathrm{U}_{n} \mathrm{c}_{1}\right) .
\end{aligned}
$$

이 식에서 2 차 섭동값들을 무시하면, (26)은 다음 과 같이 다시 쓸 수 있다.

$$
\begin{gathered}
\left(\mathrm{W}_{L}^{H} \mathrm{QW} \mathrm{W}_{L}\right) \mathrm{u}_{s}+\left(\mathrm{W}_{L}^{H} \Delta \mathrm{QW}_{L}\right) \mathrm{u}_{s}+\left(\mathrm{W}_{L}^{H} \mathrm{QW} \mathrm{W}_{L}\right) \mathrm{U}_{n} \mathrm{c}_{1} \\
=\Sigma_{s} \mathrm{u}_{s}+\Delta \Sigma_{s} \mathrm{u}_{s}+\Sigma_{s} \mathrm{U}_{n} \mathrm{c}_{1} .
\end{gathered}
$$

이제, 관계식 $\quad\left(\mathrm{W}_{L}^{H} \mathrm{QW}_{L}\right) \mathrm{u}_{s}=\mathrm{Su}_{s}=\Sigma_{s} \mathrm{u}_{s}$ 과 $\left(\mathrm{W}_{L}^{H} \mathrm{QW}_{L}\right) \mathrm{U}_{n} \mathrm{c}_{1}=\mathrm{SU}_{n} \mathrm{c}_{1}=\mathrm{U}_{n} \Sigma_{n} \mathrm{c}_{1}$ 을 이용하면, (27)은 다음과 같이 쓸 수 있다.

$\left(\mathrm{W}_{L}^{H} \Delta \mathrm{QW}_{L}\right) \mathrm{u}_{s}+\mathrm{U}_{n} \Sigma_{n} \mathrm{c}_{1}=\Delta \Sigma_{s} \mathrm{u}_{s}+\Sigma_{s} \mathrm{U}_{n} \mathrm{c}_{1}$.

이 식의 양변의 왼쪽에 $\mathrm{U}_{n}^{H}$ 을 곱하면, (28)은 다 음과 같이 쓸 수 있다.

$$
\mathrm{U}_{n}^{H}\left(\mathrm{~W}_{L}^{H} \Delta \mathrm{QW}_{L}\right) \mathrm{u}_{s}+\Sigma_{n} \mathrm{c}_{1}=\Sigma_{s} \mathrm{c}_{1} .
$$

$\Sigma_{s}=1+\tilde{\sigma}_{z}^{2}$ 이고 $\Sigma_{n}=\tilde{\sigma}_{z}^{2} \mathrm{I}_{L-1}$ 이므로, 다음 식을 얻는다.

$$
\mathrm{c}_{1}=\mathrm{U}_{n}^{H}\left(\mathrm{~W}_{L}^{H} \Delta \mathrm{Q} \mathrm{W}_{L}\right) \mathrm{u}_{s} .
$$

그리고 $\mathrm{u}_{s}=\mathrm{g}$ 이므로, $\mathrm{c}_{1}$ 은 다음과 같이 쓸 수 있 다.

$$
\mathrm{c}_{1}=\mathrm{U}_{n}^{H}\left(\mathrm{~W}_{L}^{H} \Delta \mathrm{QW} \mathrm{W}_{L}\right) \mathrm{g} .
$$


최종적으로, 다음 식을 얻는다.

$$
\Delta \mathrm{g}=\Delta \mathrm{u}_{s}=\mathrm{U}_{n} \mathrm{U}_{n}^{H}\left(\mathrm{~W}_{L}^{H} \Delta \mathrm{QW} \mathrm{W}_{L}\right) \mathrm{g}
$$

다음 제안 명제 2 는 본 논문에서 제안하는 채널 추정 방식에 대한 이론적인 채널 추정 오차 성능을 나타낸다.

(제안 명제 2) $M$ 이 충분히 큰 경우에, 제안하는 채널 추정 방식의 MSE는 다음과 같다.

$$
\begin{aligned}
\mathrm{MSE} & =E\left[\|\hat{\mathbf{g}}-\mathrm{g}\|^{2}\right]=E\left[\|\Delta \mathrm{g}\|^{2}\right] \\
& =\operatorname{tr}\left\{\mathrm{CW}_{L} \mathrm{U}_{n} \mathrm{U}_{n}^{H} \mathrm{~W}_{L}^{H}\right\} .
\end{aligned}
$$

여기에서,

$$
[\mathrm{C}]_{i, j}=\frac{1}{M}[\mathrm{R}]_{i, j} \sum_{m=1 l=1}^{N} \sum_{l=1}^{N} \frac{1}{[\mathrm{~B}]_{i, m}[\mathrm{~B}]_{j, l}^{*}} H_{l}^{*}[\mathrm{R}]_{l, m} H_{m}
$$

(증명) 제안 명제 1 을 이용하면 제안하는 채널 추정 방식의 MSE는 다음과 같이 쓸 수 있다.

$$
\begin{aligned}
\mathrm{MSE} & =E\left[\|\Delta \mathrm{g}\|^{2}\right]=E\left[\left\|\mathrm{U}_{n} \mathrm{U}_{n}^{H} \mathrm{~W}_{L}^{H} \Delta \mathrm{Qh}\right\|^{2}\right] \\
& =E\left[\operatorname{tr}\left\{\mathrm{U}_{n} \mathrm{U}_{n}^{H} \mathrm{~W}_{L}^{H} \Delta \mathrm{Qhh}^{H} \Delta \mathrm{Q}^{H} \mathrm{~W}_{L} \mathrm{U}_{n} \mathrm{U}_{n}^{H}\right\}\right] \\
& =\operatorname{tr}\left\{\mathrm{U}_{n} \mathrm{U}_{n}^{H} \mathrm{~W}_{L}^{H} E\left[\Delta \mathrm{Qhh}^{H} \Delta \mathrm{Q}^{H}\right] \mathrm{W}_{L} \mathrm{U}_{n} \mathrm{U}_{n}^{H}\right\} \\
& =\operatorname{tr}\left\{E\left[\Delta \mathrm{Qhh}^{H} \Delta \mathrm{Q}^{H}\right] \mathrm{W}_{L} \mathrm{U}_{n} \mathrm{U}_{n}^{H} \mathrm{U}_{n} \mathrm{U}_{n}^{H} \mathrm{~W}_{L}^{H}\right\} \\
& =\operatorname{tr}\left\{E\left[\Delta \mathrm{Qhh}^{H} \Delta \mathrm{Q}^{H}\right] \mathrm{W}_{L} \mathrm{U}_{n} \mathrm{U}_{n}^{H} \mathrm{~W}_{L}^{H}\right\} \\
& =\operatorname{tr}\left\{\mathrm{CW}_{L} \mathrm{U}_{n} \mathrm{U}_{n}^{H} \mathrm{~W}_{L}^{H}\right\} .
\end{aligned}
$$

여기에서 $C=E\left[\Delta \mathrm{Qhh}^{H} \Delta \mathrm{Q}^{H}\right]$ 이고, $\mathrm{h}=\mathrm{W}_{L} \mathrm{~g}$ 이 다. 이제 다음과 같이 $\Delta \mathrm{Q}$ 를 정의한다.

$$
\Delta \mathrm{Q}=\left[\begin{array}{cccc}
\Delta Q_{1,1} & \Delta Q_{1,2} & \cdots & \Delta Q_{1, N} \\
\Delta Q_{2,1} & \Delta Q_{2,2} & \cdots & \Delta Q_{2, N} \\
\vdots & \vdots & \ddots & \vdots \\
\Delta Q_{N, 1} & \Delta Q_{N, 2} & \cdots & \Delta Q_{N, N}
\end{array}\right]=\left[\begin{array}{c}
\Delta \mathrm{q}_{1}^{H} \\
\Delta \mathrm{q}_{2}^{H} \\
\vdots \\
\Delta \mathrm{q}_{N}^{H}
\end{array}\right]
$$

그러면, C는 다음과 같이 쓸 수 있다.

$$
\mathrm{C}=E\left\{\left[\begin{array}{c}
\Delta \mathrm{q}_{1}^{T} \mathrm{~h} \\
\Delta \mathrm{q}_{2}^{T} \mathrm{~h} \\
\vdots \\
\Delta \mathrm{q}_{N}^{T} \mathrm{~h}
\end{array}\right]\left[\mathrm{h}^{H} \Delta \mathrm{q}_{1}^{*}, \mathrm{~h}^{H} \Delta \mathrm{q}_{2}^{*}, \cdots, \mathrm{h}^{H} \Delta \mathrm{q}_{N}^{*}\right]\right\}
$$

따라서, $\mathrm{C}$ 의 $(i, j)$ 번째 원소는 다음과 같이 주어 진다.

$$
\begin{aligned}
{[\mathrm{C}]_{i, j} } & =E\left[\left(\Delta \mathrm{q}_{i}^{T} \mathrm{~h}\right)\left(\mathrm{h}^{H} \Delta \mathrm{q}_{j}^{*}\right)\right] \\
& =E\left[\mathrm{~h}^{H} \Delta \mathbf{q}_{j}^{*} \Delta \mathbf{q}_{i}^{T} \mathrm{~h}\right]=\mathrm{h}^{H} E\left[\Delta \mathrm{q}_{j}^{*} \Delta \mathbf{q}_{i}^{T}\right] \mathrm{h} \\
& =\mathrm{h}^{H} \mathrm{~T}^{(j, i)} \mathrm{h} .
\end{aligned}
$$

여기에서, $\mathrm{T}^{(j, i)}$ 는 다음과 같다.

$$
\begin{aligned}
\mathrm{T}^{(j, i)} & =E\left[\Delta \mathrm{q}_{j}^{*} \Delta \mathrm{q}_{i}^{T}\right] \\
& =E\left\{\left[\begin{array}{c}
\Delta Q_{j, 1}^{*} \\
\Delta Q_{j, 2}^{*} \\
\vdots \\
\Delta Q_{j, N}^{*}
\end{array}\right]\left[\begin{array}{ll}
\Delta Q_{i, 1} \Delta Q_{i, 2} \cdots \Delta Q_{i, N}
\end{array}\right]\right\} .
\end{aligned}
$$

$\mathrm{T}^{(j, i)}$ 의 $(l, m)$ 번째 원소는 다음과 같이 주어진다.

$$
\left[\mathrm{T}^{(j, i)}\right]_{l, m}=E\left[\Delta Q_{j, l}^{*} \Delta Q_{i, m}\right] .
$$

$\hat{\mathrm{Q}}=\hat{\mathrm{R}} / \mathrm{B}$ 이기 때문에, $\quad \hat{Q}_{m, n}=\hat{R}_{m, n} / B_{m, n}$ 과 $\Delta \hat{Q}_{m, n}=\Delta \hat{R}_{m, n} / B_{m, n}$ 을 얻는다. 따라서, $\left[\mathrm{T}^{(j, i)}\right]_{l, m}$ 는 다음과 같이 쓸 수 있다.

$$
\left[\mathrm{T}^{(j, i)}\right]_{l, m}=\frac{1}{B_{i, m} B_{j, l}^{*}} E\left[\Delta \widehat{R}_{i, m} \Delta \hat{R}_{j, l}^{*}\right]
$$

[9]에 의해, $E\left[\Delta \hat{R}_{i, m} \Delta \hat{R}_{j, l}^{*}\right]=R_{i, j} R_{l, m} / M$ 이기 때 문에, $\left[\mathrm{T}^{(j, i)}\right]_{l, m}$ 는 다음과 같이 쓸 수 있다.

$$
\left[\mathrm{T}^{(j, i)}\right]_{l, m}=\frac{1}{M} \frac{1}{B_{i, m} B_{j, l}^{*}} R_{i, j} R_{l, m}
$$

이제 (38)은 다음과 같이 다시 쓸 수 있다.

$$
\begin{aligned}
{[\mathrm{C}]_{i, j} } & =\mathrm{h}^{H} \mathrm{~T}^{(j, i)} \mathrm{h}=\sum_{m=1}^{N}\left(\sum_{l=1}^{N} H_{l}^{*}\left[\mathrm{~T}^{(j, i)}\right]_{l, m}\right) H_{m} \\
& =\sum_{m=1}^{N}\left\{\sum_{l=1}^{N} H_{l}^{*} \frac{1}{B_{i, m} B_{j, l}^{*}} \frac{1}{M} R_{i, j} R_{l, m}\right\} H_{m} \\
& =\frac{1}{M} R_{i, j} \sum_{m=1 l=1}^{N} \sum_{i}^{N} \frac{1}{B_{i, m} B_{j, l}^{*}} H_{l}^{*} R_{l, m} H_{m} .
\end{aligned}
$$

\section{$\mathrm{V}$. 모의실험 및 성능 분석}

본 장에서는 모의실험을 통하여 제안한 알고리듬 
의 성능을 확인한다. 다음과 같이 지수 전력 지연 분포 특성을 갖는 채널 모델을 사용한다 ${ }^{[4]}$.

$$
E\left\{\left|g_{l}\right|^{2}\right\}=\exp (-l / 10), l=0,1, \cdots, L-1
$$

여기에서, $L$ 은 채널 탭의 개수로서, [4]에서처럼 $L=3$ 이라고 가정하고, 각 채널 탭 경로의 위상은 $[0,2 \pi\}$ 에서 균일하게 분포한다고 가정한다. 부반송 파의 개수는 $N=32$ 를 사용한다. 송신 심볼들의 신 호점은 QPSK (Quaternary phase-shift keying)를 사 용한다. 모든 미상 채널 추정기에서와 마찬가지로 제안하는 채널 추정 방법도 추정된 채널 벡터에 위 상 모호성이 존재한다. 이것을 해결하기 위한 훈련 심볼을 전송하지 않고, 단순히 모의실험을 위한 목 적으로 다음과 같이 위상 모호성을 제거한다 ${ }^{[4]}$.

$$
\alpha_{0}=\arg \min _{\alpha}\|\mathbf{g}-\alpha \hat{\mathbf{g}}\|^{2} .
$$

이제 채널 추정 방법들에 대한 MSE 추정치는 다음과 같이 정의한다.

$$
\mathrm{MSE}_{0}=\frac{1}{J} \sum_{i=1}^{J}\left\|\mathbf{g}-\alpha_{0} \hat{\mathbf{g}}(i)\right\|^{2}
$$

여기에서 $i$ 는 $i$ 번째 모의실험 수행을 나타내며, $J=500$ 번의 독립적인 모의실험 수행 결과를 평균 한 값이다.

수학식 (5)와 (6)에서 제시된 두 가지 특성을 만 족시키는 행렬 $\mathrm{P}$ 는 [3]에서 주어진 것과 같이 선택 할 수 있으며, 다음과 같다.

$$
[\mathrm{P}]_{m, n}= \begin{cases}1 / \mu, & n=m \neq K \\ j(-1)^{n} A / \mu, & n=K \neq m \\ \left\{1+j(-1)^{n} A\right\} / \mu, & n=m=K \\ 0, & \text { otherwise. }\end{cases}
$$

여기에서 $K$ 는 $[1, N]$ 에 존재하는 자연수 값이고, $\mu$ 는 $\mu=\sqrt{1+A^{2}}$ 이고, $A$ 는 $|A|<1$ 인 임의의 실 수 값으로서, 본 모의실험에서는 $A=0.2$ 를 사용하 였다.

그림 1 은 $\mathrm{SNR}$ 에 따른 채널 추정 $\mathrm{MSE}$ 성능을 나타낸다. 이 그림에서 'Proposed (Ana)'로 표시된 그래프는 제안한 채널 추정 방법에 대한 이론적 MSE 성능 값으로서 (32)를 이용하여 구한 성능이 고, 'Proposed $(\mathrm{Sim})$ '은 모의실험을 통해 구한 성능

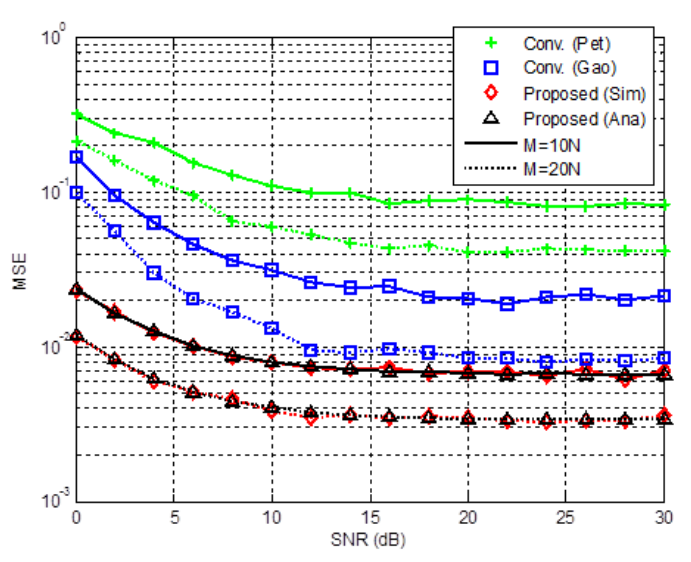

그림 1. 제안하는 채널 추정 방법과 기존 방법들 간의 MSE 비교

Fig. 1. MSE comparison between the proposed and conventional channel estimation schemes

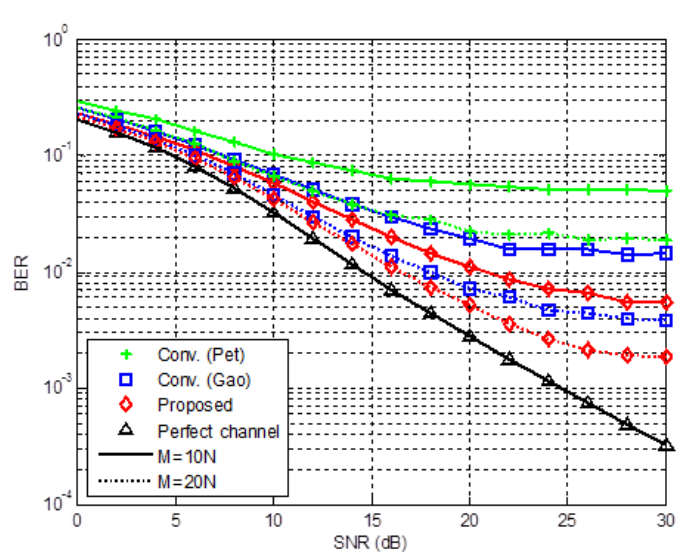

그림 2 제안하는 방법과 기존 방법들 간의 $\mathrm{BER}$ 성능 비교 Fig. 2 BER comparison between the proposed and conventional schemes

이다. 그리고, 'Conv. (Pet)'와 'Conv. (Gao)'는 기 존 방법들로서, 각각 [3]과 [4]에 대한 성능을 나타 낸다. 실선으로 표시한 그래프는 식 (13)의 $M$ 값에 대해서 $M=10 N$ 을 사용한 경우에 대한 결과들이 고, 점선으로 표시한 그래프는 $M=20 N$ 인 경우에 대한 결과들이다. 이 그림으로부터, 이론적으로 구 한 채널 추정 MSE 값이 모의실험으로 구한 값과 일치한다는 것을 알 수 있다. 그리고, 제안하는 방 법이 기존 방법들보다 더 우수한 채널 추정 성능을 보인다는 것을 알 수 있다. 또한, $M$ 값이 증가할수 록 모든 방식들의 채널 추정 성능이 좋아진다는 것 을 알 수 있으며, 이것은 수신 신호를 이용하여 추 정한 공분산 행렬이 점점 더 정확해지기 때문이다.

그림 2는 SNR에 따른 BER (Bit error rate) 성 
능을 나타낸다. 전송 심볼을 추정하기 위하여 MMSE (Minimum mean square error) 수신기 [4] 를 사용하였다. 이 때, 각각의 채널 추정 방식으로 얻은 채널 값들을 이용하여 MMSE 수신기를 설계 하였다. 그림에서, 'Perfect channel'은 채널 추정이 완벽한 경우에 대한 결과를 나타낸다. 이 그림으로 부터, 제안하는 채널 추정 방식을 적용한 수신기의 $\mathrm{BER}$ 성능이 기존 방법들보다 더 우수하다는 것을 알 수 있으며, $M$ 값이 증가할수록 $\mathrm{BER}$ 성능이 좋 아진다는 것을 알 수 있다.

\section{VI. 결 론}

본 논문에서는 OFDM 시스템에서 비중복 프리코 딩을 이용한 미상 채널 추정 방법을 제안하였다. 제 안하는 채널 추정기의 MSE 성능을 이론적으로 구 하였으며, 이 결과가 모의실험 결과와 일치한다는 것을 보였다. 또한, 모의실험을 통해서, 제안하는 채 널 추정 방법이 기존 방법들과 비슷한 복잡성을 요 구하면서, 기존 방법들보다 더 우수한 MSE 및 $\mathrm{BER}$ 성능을 나타낸다는 것을 보였다.

\section{참 고 문 헌}

[1] Cai and A. N. Akansu, "A subspace method for blind channel identification in OFDM systems," in Proc. ICC, New Orleans, LA, July 2000, pp. 929-933.

[2] B. Muquet, M. de Courville, and P. Duhamel, "Subspace-based blind and semi-blind channel estimation for OFDM systems," IEEE Trans. Signal Process., vol. 50, pp. 1699-1712, July 2002.

[3] A. Petropulu, R. Zhang, and R. Lin, "Blind OFDM Channel Estimation Through simple Linear Precoding," IEEE Trans. Wireless Commun.,vol. 3, pp. 647-655, Mar. 2004.

[4] F. Gao and A. Nallanathan, "Blind Channel Estimation for OFDM Systems via a Generalized Precoding," IEEE Trans. Vehic. Technol., vol. 56, pp. 1155-1164, May 2007.

[5] R. Lin and A. Petropulu, "Linear Precoding Assisted Blind Channel Estimation for OFDM Systems," IEEE Trans. Vehic. Technol., vol. 54, pp. 983-995, May 2005.
[6] J. Gao, X. Zhu, and A. K. Nandi, "Non-redundant precoding and PAPR reduction in MIMO OFDM systems with ICA based blind equalization," IEEE Trans. Wireless Commun., vol. 8, pp. 3038-3049, June 2009.

[7] J. J. Murillo-Fuentes and R. Boloix-Tortosa, "Strict separability and identifiability of a class of ICA models," IEEE Trans. Signal Process. Letters, vol. 17, pp. 285-288, Mar. 2010.

[8] G. H. Golub, Matrix Computation, 3rd Edition, The Johns Hopkins University Press, 1996.

[9] F. Li, H. Liu, and R. J. Vaccaro, "Performance Analysis for DOA Estimation Algorithms: Unification, Simplification, and Observations," IEEE Trans. Aero. Electro. Systems, vol. 29, pp. 1170-1184, Oct. 1993.

서 방 원 (Bangwon Seo) 정회원

1997년 2월 한국과학기술원 전

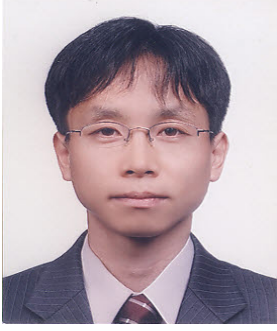

기 및 전자공학과 공학사 1999년 2월 한국과학기술원 전

기 및 전자공학과 공학석사 2010년 2월 한국과학기술원 전 기 및 전자공학과 공학박사 2004년 11월 현재 한국전자통 신연구원 선임연구원

<관심분야> 이동통신 기술, 통신 신호처리, MIMO, OFDM, CDMA, Cognitive Radio 